\title{
Clinical development of biological response modifiers
}

\author{
JAY P SIEGEL MD FACP
}

\begin{abstract}
JP SIEGEL. Clinical development of biological response modifiers. Can J Infect Dis 1994;5(Suppl A): 5A-8A.

OвJEcTIVE: To present perspectives on selected issues that frequently arise during the clinical development of biological response modifier (BRM) therapies.

Data Sources: The perspectives and opinions presented herein were developed over several years of reviewing and consulting on the clinical development of BRM therapies at the United States Food and Drug Administration.

Conclusions: BRM therapies encompass a broad spectrum of products used to treat a wide variety of diseases. Due to this diversity, most principles of clinical trial design and conduct applicable to the majority of BRMS are those that are applicable to all therapies. Nevertheless, the clinical development of BRM therapies often raises specific issues and problems in the areas of selecting animal models, defining the study population. adverse reactions, dosing and defining end-points. Over 10 years' experience in testing biotechnology derived BRMs in clinical trials has created a database from which we can draw valuable generalizations for guidance in future studies.
\end{abstract}

Key Words: Biological response modifiers, Biologicals, Clinical trials, Drug development

\section{Développement clinique des modificateurs de la réponse biologique}

RÉSUMÉ : OвJEctif: Présenter divers aspects du développement clinique des thérapeutiques à base de modificateurs de la réponse biologique.

Sources des Données: Les perspectives et les opinions présentêes ici se sont développées au cours des ans par l'analyse et la consultation de documents sur l'évolution clinique des traitements par mRB à la United States Food and Drug Administration.

Conclusions: Les traitements par mRB comprennent un large éventail de produits utilisés pour traiter une grande variété de maladies. À cause de cette diversité, la plupart des principes qui régissent les modèles d'essais cliniques et la conduite à appliquer pour la majorité des MRB sont ceux qui s'appliquent à toutes les thérapies. Néanmoins, l'évolution clinique des traitements par MRB soulève parfois des problèmes spécifiques en ce qui concerne le choix de modèles animaux, la définition des populations étudiées, les réactions indésirables, la posologie et les paramètres de déterminants. Après dix années d'expérience biotechnologique sur les MrB dans le cadre d'essais cliniques, une base de données a été créée, à partir de laquelle des généralisations utiles peuvent être formulées pour orienter les études futures.

Division of Clinical Trial Design and Analysis, Center for Biologics Evaluation and Review, Food and Drug Administration, Rockville, Maryland, USA

Correspondence and reprints: Dr Jay P Siegel, DCTDA, OTRR, CBER, FDA HFM-570, 1401 Rockville Pike, Suite 200S, Rockville, MD 20852, USA 
$\mathrm{B}$ OLOGICAL RESPONSE MODIFIER (BRM) THERAPY REFERS TO herapeutic interventions that target immunological, inflammatory or other host responses to infection, malignancy or other abnormal physiological events. The pace of development of BRM therapy has increased exponentially in recent years. The categories of BRM agents include antisera, monoclonal antibodies, cytokines, growth factors, fusion proteins, adoptive immunotherapy, somatic cell therapy, gene therapy, immunomodulating drugs and products derived from, or related to, the preceding types of products. At present, BRMs are in use or under clinical investigation for the treatment of patients with a broad variety of conditions including: malignancies; infectious diseases of viral, bacterial and fungal origin; transplantation of bone marrow and solid organs; and immunodeficiency diseases. Thus, it is difficult to generalize about the clinical development of BRMs beyond saying that general principles of good clinical trial design, conduct and analysis should be applied.

Nevertheless, the property of being a BRM has important implications for all aspects of clinical development. Most notable are the selection of animal models, defining the study population, evaluating adverse reactions, dosing and defining end-points. Additionally, BRMs that are proteins, living cells and/or analogues of endogenous substances raise additional issues in clinical development as a result of these properties.

\section{ANIMAL MODELS}

Although testing in animal models is not actually a clinical trial, animal testing is so crucial to clinical development that it is worthwhile to consider briefly some key issues in this context. BRMs can present many difficulties in animal testing, particularly as a result of species restriction and immunogenicity. A BRM may be species restricted as a result of absent or decreased affinity of the homologous receptor (eg, a cytokine such as granulocyte-macrophage colony-simulating factor [GM-CSF] or interferon [IFN]- $\gamma$ ], absence or alteration of the target antigen (eg, for a monoclonal antibody) and/or major histocompatibility complex restriction (eg, for a lymphocyte clone). Interpretation of long term studies of protein BRMs may be complicated by the formation of antibodies altering pharmacokinetics and/or pharmacodynamics. Cellular therapies may be subject to lack of activity due to species restriction and/or altered survival and function resulting from xenogeneic cellular and humoral immune responses.

There is relatively little information to be gained from studying species restricted BRMs in species in which they lack BRM activity. Such studies may yield some information regarding reactions due to impurities and reactions unrelated to the intended mode of action. However, since many BRMS are quite pure, and since most species restricted BRMs act at low concentrations through high affinity receptors or ligands, the most important adverse and desired reactions to these therapies in humans are almost invariably related to specific binding. Such effects can only be studied in species in which binding and biological activity of the BRM occur.

Due to the difficulties in animal testing of BRMS, many are introduced into clinical trials with little relevant animal data. The resultant paucity of information regarding beneficial and toxic effects, therapeutic and toxic doses, and effects of various doses, routes, schedules and combinations on the desired BRM effect requires that clinical studies proceed in a slow and cautious manner to ensure safety. Thus, the starting dose will be low, escalation will be slow and obtaining answers to key pharmacological questions will be slow, costly, inefficient and sometimes dangerous. While such clinical difficulties may at times be unavoidable, their undesirability provides a strong impetus for testing BRMs in an appropriate animal model where possible. For species restricted BRMs, production and testing of the homologous animal product, while not part of the classic toxicological testing, may provide the most valuable information about the likely pharmacological effects of the human analogue. For many relatively species restricted products, responses in primate species may adequately reflect human responses. Additionally, animals engineered to be responsive to the BRM, eg, transgenic animals expressing the relevant human antigen or receptor, athymic nude mice or severe combined immunodeficiency disease mice bearing BRM responsive human immune cells may provide useful information, but only to the extent that the model reflects human conditions.

It should be noted that even BRMs that act across species lines may show marked interspecies variation in dose response profiles or nature of effect. The former may result from differences in the affinity of the receptors or from other differences in the sensitivity of the species tested in evoking the measured biological response. For these reasons, standard approaches used for extrapolation of doses to humans, eg, body surface area, weight and allometric scaling, may not be reliable. Information about the relative dose response and binding affinities of the cells of the test species and of humans can be of great value in extrapolating dosage from the test species to humans. Such information should be generated before clinical testing whenever possible.

\section{THE STUDY POPULATION}

In traditional drug development, testing in normal volunteers can be an extremely valuable early source of information about the pharmacology of a drug. This type of testing is far less common in the development of BRMs, largely because the potential risk to humans of a new BRM is rarely well characterized before testing in humans. Even when good animal models are available and species restriction is not a problem, some key 
questions, notably the degree and the clinical implications of immunogenicity of a human protein in humans, cannot be well assessed in animals. Where an unknown but significant possibility of substantial harm to the subject exists, testing on normal volunteers with no potential for direct benefit is problematic. To keep risks and benefits in an appropriate balance, BRMs are often first tested in humans with a disease that might benefit from therapy, frequently an ultimately fatal disease.

The target population for most BRMs includes immunocompromised patients, such as patients with advanced malignancies or immunodeficiency disorders, transplant recipients, recipients of myelotoxic therapies, neonates and the elderly. In studying BRMs in immunosuppressed patients it is important to bear in mind that the effects of many BRMs, both desired and undesired, may be mediated by cells of the immune system. Patients in whom the target cells of the BRM are either absent or irreversibly hypofunctional may not respond to a BRM as would other individuals. In general, the optimal target population for early trials of a BRM therapy consists of patients who have a serious or terminal disease that might benefit from the therapy, but in whom physiological functions, particularly drug metabolic and excretory functions, immunological functions and the ability to tolerate drug toxicities, are relatively intact.

\section{ADVERSE REACTIONS}

BRMS are capable of inducing a broad range of adverse reactions. Nevertheless, a number of generalizations can be made regarding adverse reactions to BRMs, and they should be born in mind in planning clinical development.

First, certain patterns of toxicity appear to be particularly common in BRM therapy. Central nervous system toxicities include agitation, seizure, lethargy, coma with interleukin (IL)-2 and mood changes with IFN- $\alpha$. Vascular leak leading to effusions and edema have been observed with IL-2, GM-CSF and tumour necrosis factor (TNF) $-\alpha$. Although the endocrinological effects of many cytokines have yet to be fully investigated, $\mathrm{L}-2$ therapy has been reported to be associated with elevated levels of corticotropin, cortisol, prolactin, growth hormone and beta-endorphin and decreased levels of thyroid hormone, while IFN- $\alpha$ therapy has been reported to be associated with lowered serum estradiol and progesterone levels (as well as menstrual irregularities, decreased fertility and increased spontaneous abortions in primates). Perhaps these patterns are not surprising in that there are increasingly recognized close interactions between the immune system and the neurological and endocrine systems, and because regulation of vascular permeability is critical to regulation of immunological and inflammatory responses. Other common toxicities of immunomodulating BRMs include fever and 'flu-like symptoms observed with a variety of cytokines including IFN- $\alpha$, IFN- $\beta$, IL- 2 , TNF- $\alpha$ and GM-CSF. The similarity of toxicities observed with a variety of immunoregulatory cytokines and other BRMs should not be surprising in that cytokines are involved in a complex regulatory network in which there is considerable functional overlap; the administration of one cytokine often induces the production of several others cytokines.

Second, many BRM therapies have significant theoretical possibility of exacerbating disease. Exacerbation may occur through several mechanisms. Host defences are not only responsible for control of malignancies and infections, but they may also cause pathology. Since the physiological regulation of host defences is poorly understood, attempts to improve natural defences, regardless of the effector mechanism targeted, may adversely affect disease control and/or may contribute to tissue destruction or dysfunction associated with the disease. Thus, preliminary data in sepsis trials suggest some anti-inflammatory BRMs may be associated with higher mortality rates when administered to some patients with sepsis. In malignancies in which the tumour cells may bear receptors for cytokines and growth factors employed in or induced by BRM therapy, (eg, many hematological malignancies), the possibility that the BRM will promote growth of the tumour must be considered.

Third, BRM therapy may lead to the production of antibodies. BRMs that are proteins are often immunogenic. Even those proteins that are very closely related to endogenous substances may be immunogenic due to the unusual dose, schedule and route of presentation to the immune system. Antibody formation is particularly likely after repeated, prolonged and intermittent administration, and should be assessed for all protein BRMs. When antibodies are detected they should be tested for their ability to neutralize the BRM and, where relevant, its natural analogue. Furthermore, it is important to study patients developing antibody reactions for the following potential consequences: loss of response (therapeutic or adverse) to the BRM; change in pharmacokinetics of the BRM; immune complex disease; and loss of activity of the endogenous natural analogue of the BRM. Information on the duration of the antibody response and, when they can be obtained safely, data regarding the effects of readministration can be of great value. In addition to the immunogenicity of proteins, the immunogenicity of cellular therapies, whether modified autologous, allogeneic or xenogeneic cells, is a major concern under active study.

\section{DOSING}

As noted above, performing studies in a relevant animal model, and determining the quantitative relationship of dose response effects on animal and human cells, can be of great value in determining a safe starting dose and escalation rate that will allow early study 
of biologically active doses. Determining the optimal dose (as well as route and schedule) of a BRM to carry forward into further phase 2 and 3 trials can be difficult. Typically, chemotherapeutic agents for the treatment of malignancies or serious infections are tested and ultimately used at maximally tolerated doses. In the area of BRM therapies, it has long been argued that more is not always better and that the optimal dose of a BRM is the lowest dose causing a maximal modification of targeted biological response.

Determining an optimal biological response modifying dose requires having a relevant, valid and reproducible assay of the biological response under consideration or a closely related biological effect. Functional assays of cells are notoriously difficult to make standard and reproducible, particularly in multicentre studies where multiple laboratories or transport of living cells must be used. Where possible, biological effects should be measured by cell counts, serum proteins or chemistries, or chemical or molecular rather than functional studies on cells. Considerable efforts should go into standardizing and ensuring reproducibility of these assays.

Additionally, it is important to ensure that any biological effect being used to guide dosing or other key aspects of drug development be relevant. Although it is desirable that the biological effect assayed be the response that is hypothesized to cause clinical improvement, this is not always feasible. In such cases, one should ensure that the monitored biological effect depends on the same receptor and, preferably, the same effector cell, as the targeted response.

\section{END-POINTS}

As noted in the section on dosing, measurement of the biological effects of a BRM can provide an end-point of great value in the clinical testing of a BRM. Such effects, if reliably measured and related to clinical outcome, can be used to optimize dose, route, schedules, combination therapy and target population. Of course, determinations of efficacy of a BRM must be based on end-points that reflect meaningful changes in disease course or symptoms. Beginning with the early trials of a BRM, one should put considerable efforts into correlating measured biological effects with these clinically significant events. Evidence of such correlations can help select which biological effect to optimize in future drug development and may allow use of early biological effect measurements to identify those patients most likely to benefit from ongoing therapy. When the biological effect can be measured reproducibly and the correlation with clinical events is strong and consistent, it may be valid to use the biological response measurement as a surrogate for clinical efficacy, thus greatly facilitating the clinical investigation of the BRM and closely related therapies.

\section{SUMMARY}

The clinical investigation of BRMs has been underway for many years and has accelerated recently in parallel with advances in biotechnology. The following generalizations can be made regarding the clinical development of BRM therapies:

- Animal models for testing BRMs are often difficult and costly to develop and validate. Nevertheless, such efforts can be extremely worthwhile in facilitating and expediting clinical testing.

- While the initial human testing of BRMs is often most appropriately done in patient populations, it is generally desirable that these patients have relatively intact physiological functions.

- Certain classes of BRMs induce characteristic types of adverse reactions that should be studied carefully.

- A reliable, reproducible measure of the intended biological response modification or a related biological effect can be of great value in drug development, particularly in selecting an optimal dose and regimen to evaluate for clinical efficacy.

- Studies of the relationship between measurable biological effects induced by a BRM and clinical benefit should be actively pursued throughout clinical testing. 


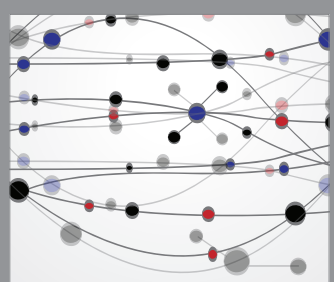

The Scientific World Journal
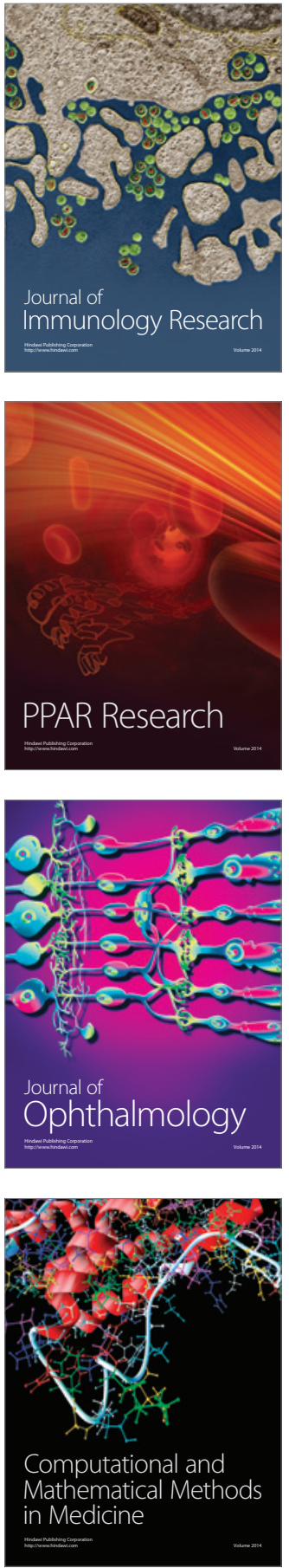

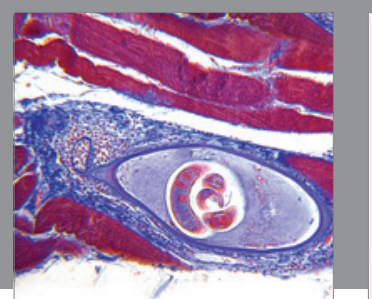

Gastroenterology Research and Practice

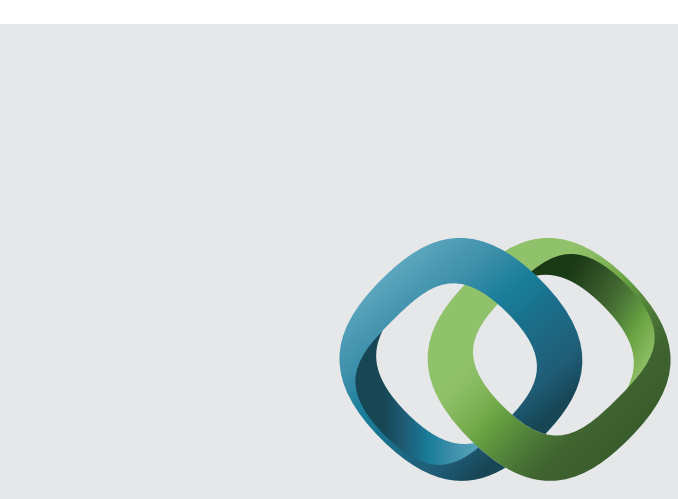

\section{Hindawi}

Submit your manuscripts at

http://www.hindawi.com
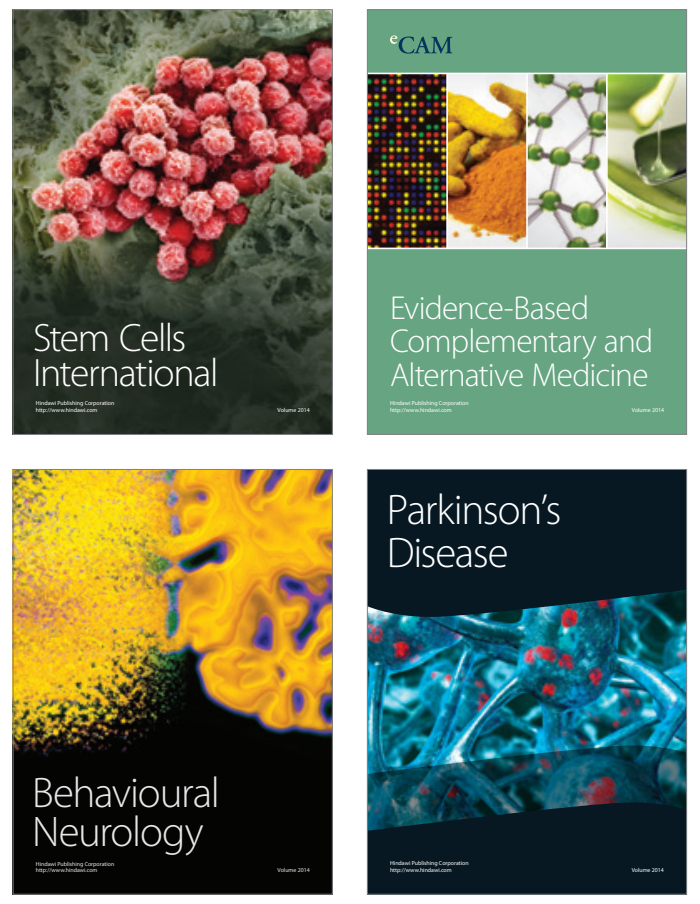
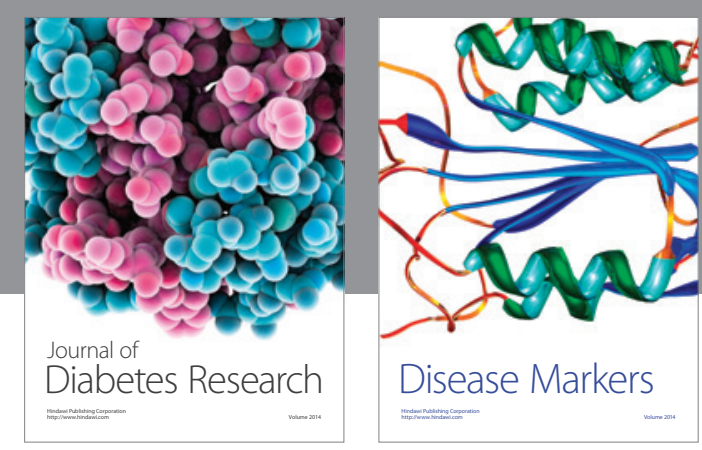

Disease Markers
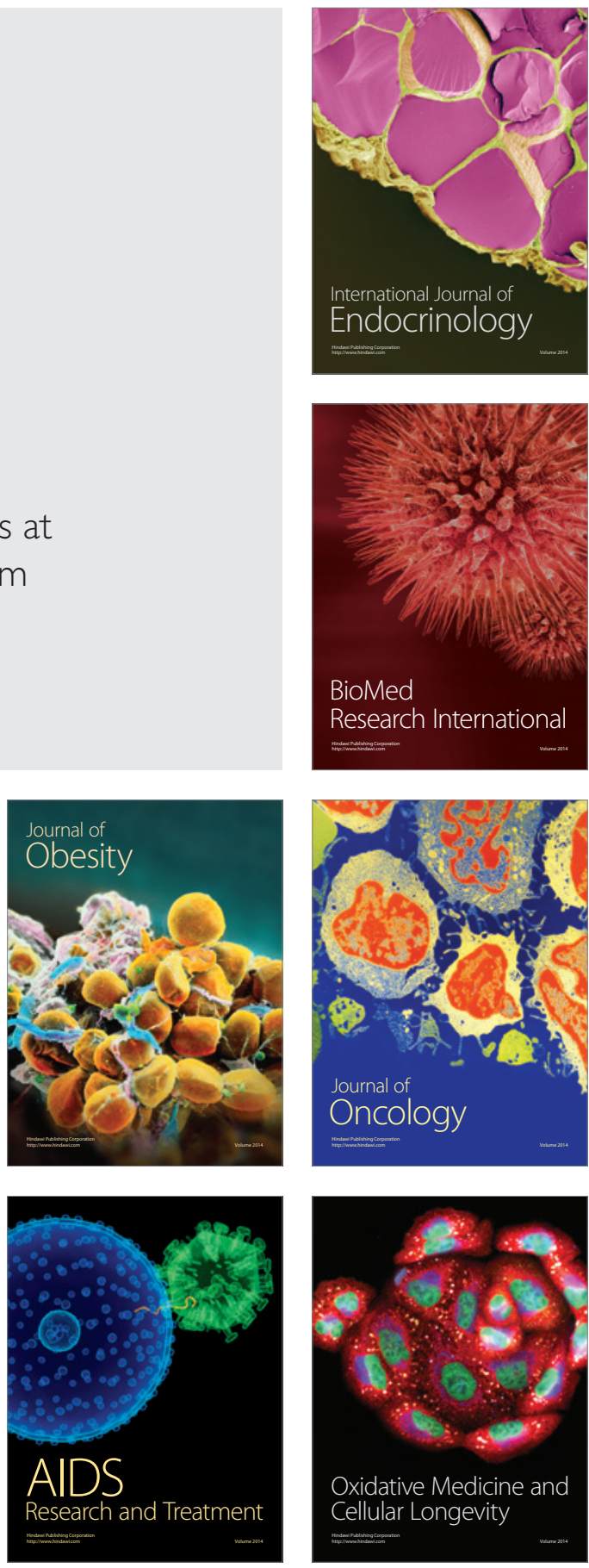